\title{
Opiniones y hábitos de publicación en acceso abierto de los investigadores argentinos. Un estudio basado en los datos de la encuesta SOAP
}

\author{
Paola Bongiovani*, Nancy Diana Gómez ${ }^{\star \star}$, Sandra Miguel**
}

\begin{abstract}
Resumen: El presente trabajo tiene como principal objetivo conocer las opiniones y hábitos de publicación en acceso abierto de los investigadores argentinos en cuatro áreas temáticas: Medicina, Física y Astronomía, Agricultura y Ciencias Biológicas y Ciencias Sociales y Humanidades. El estudio se basa en las respuestas de los investigadores que participaron de la encuesta mundial realizada en 2010 en el marco del proyecto SOAP (Study of Open Access Publishing). Los resultados obtenidos son comparados con los hallazgos de la encuesta SOAP a escala mundial, y con un estudio previo sobre prácticas de publicación de los investigadores argentinos según el modelo económico de las revistas donde publican. Las conclusiones y discusión advierten que si bien la publicación en acceso abierto es, desde las opiniones de los investigadores, considerado beneficioso, no es suficiente para que modifiquen sus hábitos de publicación.
\end{abstract}

Palabras clave: Acceso abierto, encuesta de opinión, investigadores, Argentina.

\section{Argentinian researchers' opinions and habits regarding open access publishing. A study based on the SOAP survey data}

Abstract: The aim of present work attempts was to discover the opinions and habits of Argentinean researchers regarding open access publishing in four research fields: Medicine, Physics and Astronomy, Agriculture and Biological Sciences and Social Sciences and Humanities. The study is based on the researchers' responses to the SOAP (Study of Open Access Publishing) survey conducted in 2010 and these results are compared with those revealed in the worldwide SOAP study as well as with the findings of a previous study on the publication patterns of Argentina's scientific community. The conclusions and discussion caution that while open access publishing is considered beneficial, that consideration is not sufficient in itself for researchers to change their publication habits.

Keywords: Open access, survey, researchers, Argentina.

* Centro Internacional Franco Argentino de Ciencias de la Información y de Sistemas (CIFASIS). Universidad Nacional de Rosario. Argentina. Correo-e: pbongio@unr.edu.ar.

**k Departamento de Biblioteconomía y Documentación. Facultad de Humanidades, Comunicación y Documentación. Universidad Carlos III de Madrid, España. Correo-e: ndgomez@bib.uc3m.es.

**** Instituto de Investigaciones en Humanidades y Ciencias Sociales y Departamento de Bibliotecología. Facultad de Humanidades y Ciencias de la Educación. Universidad Nacional de La Plata, Argentina. Grupo de Investigación SCImago, CSIC, Madrid, España (www.scimago.es). Correo-e: sandra@fcnym.unlp.edu.ar.

Recibido: 03-11-2011; 2. ${ }^{\mathrm{a}}$ versión: 19-12-2011; aceptado: 9-01-2012. 


\section{Introducción}

El movimiento de acceso abierto (AA) a la literatura científica es una iniciativa que propone el acceso libre y gratuito a los conocimientos que genera la ciencia. Uno de los principios básicos del AA es permitir a los usuarios la lectura, descarga, copia, distribución, impresión, búsqueda o enlace a los textos completos de los artículos, sin otras barreras económicas, legales o técnicas que las que suponga Internet en sí misma.

La Declaración del Budapest Open Access Initiative (BOAI) de 2001 constituye un hito para el desarrollo de este movimiento, porque allí se establecen las bases de las dos vías más difundidas del AA: la vía dorada y la vía verde. La primera propone la publicación de artículos en revistas de acceso abierto, teniendo el lector acceso libre y gratuito a los textos completos de los mismos. La segunda alternativa, en cambio, conocida como la vía verde, propone el auto-archivo de los artículos en repositorios institucionales o temáticos de acceso abierto, ya sea antes (pre-print) o después (post-print) de su publicación. Por tanto, se trata de dos estrategias bien diferenciadas. La vía dorada se refiere al modelo económico de las revistas, mientras que la vía verde apela a la actitud proactiva de los autores para difundir sus trabajos de investigación.

No se tienen aún certezas acerca de cuál de las dos alternativas del AA alcanzará mayor desarrollo en un futuro próximo. Algunos autores como Harnad y otros (2008) señalan que la única alternativa para alcanzar el 100\% del AA es la vía verde, debido a que no implica una reestructuración total del sistema de edición científica. Aunque se estima que hasta ahora solo el $20 \%$ de los artículos publicados en revistas que permiten auto-archivo están depositados en repositorios (Björk y otros, 2010). Por otro lado, las revistas de acceso abierto representan apenas el $10 \%$ del total de revistas científicas arbitradas de todo el mundo, aunque hay estudios que muestran que desde el año 2000 su número se viene incrementando de manera notable, a una tasa de crecimiento anual promedio del 18\% (Laakso y otros, 2011).

Un reciente informe elaborado para la Research Information Network (RIN y otros, 2011) plantea cinco escenarios posibles del desarrollo del AA en los próximos años, analizando sus costos y beneficios. El estudio sugiere que para incrementar el acceso a los resultados de investigación se debe fomentar el uso de los repositorios temáticos e institucionales existentes, a la vez que facilitar la transición a la publicación en revistas de acceso abierto, regulando los costos por publicación.

En cualquiera de los casos el éxito del movimiento AA dependerá, en gran medida, del grado de adopción de las prácticas de publicación en abierto y/o de auto-archivo en repositorios por parte de los investigadores, principales actores en el proceso de comunicación de la ciencia, así como de los acuerdos entre editores, instituciones que financian la investigación y de todas las partes involucradas en el sistema de comunicación científica.

Es por ello que el conocimiento de las opiniones y hábitos de comunicación en abierto de la comunidad científica se torna clave para identificar las tendencias y posibilidades de su desarrollo. 


\section{Revisión de la literatura}

La revisión de la literatura revela un creciente interés por conocer las opiniones y actitudes de la comunidad científica con relación a la publicación en revistas de acceso abierto.

De los estudios más recientes que muestran las opiniones a escala mundial se destaca una encuesta realizada en 2010 en el marco del proyecto SOAP (Study of Open Access Publishing). De los 53.890 científicos que participaron de dicha encuesta se analizaron las respuestas de 38.358 investigadores activos de diferentes disciplinas de 162 países (Dallmeier-Tiessen y otros, 2011).

Según se explicita en el manual de procedimiento (SOAP, 2011), la encuesta fue distribuida por correo electrónico a través de la conocida herramienta Survey Monkey. El listado de destinatarios fue construido a partir de las listas de correo electrónico de las editoriales y asociaciones participantes del consorcio SOAP (tales como SAGE, BioMed Central, Public Library of Science, Open Access Scholarly Publishers Association), y otras listas públicas con la finalidad de llegar al mayor número posible de investigadores. Se establecieron unos umbrales mínimos de 50 respuestas para cada nivel de agregación temática y de 80 respuestas por país.

Los resultados del estudio revelan que para el $89 \%$ de los investigadores el acceso abierto es considerado beneficioso para sus áreas de investigación, siendo más alto el porcentaje en Ciencias Sociales y Humanas que en otros campos. Entre las principales razones se encuentran: mejora el modo de trabajo de la comunidad científica (36\%); ofrece un mejor modelo económico financiero para la comunicación científica (20\%), y es una alternativa relevante para la consecución del bien común (20\%). Por otra parte el $71 \%$ de los investigadores señala haber publicado en abierto en los últimos cinco años, y del 29\% restante cerca de la mitad expresa no tener razones para no hacerlo. Sin embargo, un 39\% y $30 \%$, respectivamente, indican como principales barreras del AA el costo por publicación y la baja calidad de las revistas (Dallmeier-Tiessen y otros, 2011). Estos dos últimos aspectos aparecen señalados también como los principales obstáculos de la vía dorada del AA en el trabajo de Fry y otros (2011).

Por otra parte, un reciente estudio realizado entre investigadores de la European Association for Cancer Research muestra que el prestigio y el factor de impacto de las revistas son dos de los aspectos más valorados por la comunidad científica a la hora de publicar, mientras el acceso abierto tiene una importancia relativa menor (Kenney, y Warden, 2011). Hallazgos similares fueron señalados por Harley y otros (2010) a partir de los resultados de una encuesta realizada entre académicos de 45 universidades norteamericanas en siete disciplinas. El estudio concluye que los dos principales factores que influyen en la elección del medio donde publicar son el prestigio de las revistas y la adecuación de la audiencia a la que los investigadores quieren dirigir sus trabajos.

Un estudio anterior realizado por Mann y otros (2009) muestra que el 60\% de los investigadores considera que las revistas de acceso abierto son de baja calidad, y, por tanto, publicar en dichas revistas podría poner en riesgo la valo- 
ración positiva de sus publicaciones y las posibilidades de obtener fondos para financiar nuevas investigaciones. En este sentido Hurrell y Meijer-Kline (2011) advierten de la necesidad de conducir investigaciones sobre los conocimientos y actitudes que sobre el acceso abierto tienen los científicos y gestores que integran comités de evaluación de las actividades científicas.

En el ámbito de los países latinoamericanos también se empezaron a realizar algunos estudios de opiniones y prácticas de los investigadores con relación al AA. Gómez y otros (2008) mostraron que en Chile existe entre los científicos un nivel de conocimiento sobre las revistas AA entre medio (49\%) y alto (31\%); sin embargo, de este último grupo sólo el 18\% publica en ellas; básicamente porque el universo de revistas donde les interesa publicar sus trabajos es reducido y en su mayoría no son de acceso abierto. Resultados similares fueron encontrados por Sánchez Tarragó y Fernández Molina (2008) en una encuesta realizada a investigadores cubanos.

Para el caso argentino no se han encontrado estudios de opiniones sobre el AA, pero sí de las prácticas de publicación. Miguel y otros (2012) analizaron los artículos de autoría de investigadores argentinos publicados en revistas indizadas en SCOPUS en el período 2008-2010 en cuatro campos temáticos. Las autoras concluyen que Argentina tiene un gran potencial para liberar un importante porcentaje de su producción en abierto: un $27 \%$ por la vía dorada y un $43 \%$ por la vía verde. Sin embargo, observaron diferencias entre los campos temáticos. En Ciencias Sociales y Humanidades, el 35\% de las revistas elegidas para publicar son de acceso abierto y un porcentaje similar pertenece al grupo de acceso restringido que permite autoarchivo. En los otros tres campos temáticos estudiados, sin embargo, se observa una mayor preferencia por la publicación en revistas con permisos de autoarchivo: en Física y Astronomía (48,5\% vs. 8\% respectivamente); en Medicina (47,3\% vs 20\%), y en Agricultura y Ciencias Biológicas (38,5\% vs. 17,1\%).

Frente a estos resultados un estudio de las opiniones y actitudes de los investigadores argentinos en relación con las revistas de acceso abierto será un valioso complemento de estos hallazgos.

\section{Objetivos}

El presente trabajo tiene como principal objetivo conocer las opiniones y hábitos de publicación en revistas de acceso abierto de los investigadores argentinos en cuatro campos del conocimiento: Medicina, Física y Astronomía, Agricultura y Ciencias Biológicas y Ciencias Sociales y Humanidades.

Los resultados obtenidos son comparados con los hallazgos de estudios previos para determinar, por un lado, si las opiniones de los investigadores argentinos coinciden o se apartan de los hallazgos de la encuesta a escala mundial realizada en 2010 en el marco del proyecto SOAP (Study of Open Access Publishing) (Dallmeier-Tiessen y otros, 2011); y por otro, conocer si las opiniones guardan relación con las prácticas de publicación de acuerdo a los resultados encontrados en el estudio de Miguel y otros (2012). 


\section{Metodología}

El estudio se basa en las respuestas de 532 investigadores argentinos que participaron de la encuesta realizada entre el 28 de abril y el 18 de noviembre de 2010 en el marco del proyecto SOAP, cuyos datos están disponibles bajo licencia Creative Commons CC0 Dedicación al Dominio Público en: http://creativecommons.org/publicdomain/zero/1.0/deed.es.

Si bien el tamaño de la muestra es pequeño en relación a la cantidad total de investigadores del país, cuya cifra para la categoría equivalente a jornada completa (EJC) asciende a los 30.861 (Ministerio de Ciencia, Tecnología e Innovación Productiva, 2010), la cantidad representa a los investigadores que han contestado la encuesta SOAP, y obedece a los criterios adoptados y validados por los responsables de dicha encuesta. Pese a las limitaciones que ello pudiera tener, la principal ventaja es que permite que los resultados de este estudio sean comparables con los hallazgos a escala mundial o con los de cualquiera de los 162 países participantes.

\section{Preguntas de la encuesta}

Del total de preguntas de la encuesta (23) cuyo cuestionario completo se incluye en el anexo del trabajo de Dallmeier-Tiessen y otros (2011), se seleccionaron las siguientes:

\begin{tabular}{|c|c|}
\hline Preguntas & Valores de las respuestas \\
\hline $\begin{array}{l}\text { ¿Conoce revistas en su campo de investigación } \\
\text { que publican artículos en acceso abierto? (\# 8) }\end{array}$ & Sí / No / No tengo opinión. \\
\hline $\begin{array}{l}\text { ¿Piensa que es o sería beneficioso para su } \\
\text { campo de investigación la publicación de ar- } \\
\text { tículos en revistas de acceso abierto? (\# 9). } \\
\text { En caso de que la respuesta fuera afirmativa } \\
\text { explicitar los motivos: }\end{array}$ & $\begin{array}{l}\text { Sí / No / No tengo opinión / No me interesa. } \\
\text { - Accesibilidad (para referirse a las facilidades } \\
\text { en el acceso). } \\
\text { - Razones financieras (para determinar si el } \\
\text { acceso abierto se visualiza como un modelo } \\
\text { mejor o solución a problemas financieros). } \\
\text { - Beneficio individual (cuando publicar en } \\
\text { revistas de acceso abierto es percibido como } \\
\text { un valor que al investigador le permite ganar } \\
\text { mayor visibilidad, reconocimiento, lectores, } \\
\text { citas que en las revistas tradicionales). } \\
\text { - Bien público (cuando se percibe como un } \\
\text { beneficio para la sociedad). } \\
\text { - Beneficio para la comunidad científica } \\
\text { (cuando el beneficio se circunscribe a la } \\
\text { propia comunidad científica). } \\
\text { - Otras (cualquier otra cuestión o idea). }\end{array}$ \\
\hline
\end{tabular}




\begin{tabular}{|c|c|}
\hline Preguntas & Valores de las respuestas \\
\hline $\begin{array}{l}\text { ¿Qué factores influyen en la elección de una } \\
\text { revista donde publicar? (\# 13). }\end{array}$ & $\begin{array}{l}\text { [Para cada opción indique si es Muy importan- } \\
\text { te, Importante, Poco importante, Irrelevante]. } \\
\text { - Responde a la política de la institución. } \\
\text { - Experiencia positiva con el editor. } \\
\text { - Relevancia para la comunidad. } \\
\text { - Acceso abierto. } \\
\text { - Recomendado por colegas. } \\
\text { - Velocidad de publicación. } \\
\text { - Prestigio. } \\
\text { - Probabilidad de aceptación. } \\
\text { - Gratuidad. } \\
\text { - Política de copyright. } \\
\text { - Factor de impacto. } \\
\text { - Importancia de la revista para la promoción } \\
\text { académica. }\end{array}$ \\
\hline $\begin{array}{l}\text { ¿Aproximadamente cuántos artículos publicó } \\
\text { en acceso abierto en los últimos cinco años? } \\
(\# 15) \text {. }\end{array}$ & $\begin{array}{l}\text { 0/1; a } 5 / 6 \text {; a } 10 / 11 \text {; a } 15 / 6 \text {; a } 20 / 21 \text {; a } 50 \text {; Más } \\
\text { de } 50 . \\
\text { [Para este estudio las últimas cuatro categorías } \\
\text { se agruparon en una sola codificada como Más } \\
\text { de } 10 \text { ]. }\end{array}$ \\
\hline $\begin{array}{l}\text { ¿Tiene razones específicas para no publicar en } \\
\text { acceso abierto? (\# 16). }\end{array}$ & $\begin{array}{l}\text { Sí / No } \\
\text { [Si la respuesta es afirmativa señale algunas de } \\
\text { las siguientes razones]: } \\
\text { - Accesibilidad (mala experiencia con el ac- } \\
\text { ceso abierto). } \\
\text { - Presupuesto (pago por publicación). } \\
\text { - Hábito. } \\
\text { - Calidad de la revista. } \\
\text { - Lo haré la próxima vez (intención de publi- } \\
\text { car en abierto en su próximo artículo). } \\
\text { - Desconocimiento de revistas AA en su área. } \\
\text { - Otras razones. }\end{array}$ \\
\hline
\end{tabular}

Las respuestas de los investigadores argentinos a estas preguntas se agruparon tomando como base el campo de investigación principal declarado por los investigadores en la pregunta 2 de la encuesta general, en la que se especificaba el campo principal de investigación. Luego, se clasificaron en cuatro grandes áreas temáticas según los campos de conocimiento estudiados en el trabajo de Miguel y otros (2012), para facilitar la comparación de resultados (tabla I).

Conforme a este criterio se analizaron en total 463 respuestas, quedando fuera del estudio 69 correspondientes a los campos Arquitectura, Construcción y Planeamiento; Química; Ciencias de la Tierra y Matemática y Ciencias de la Computación. Luego, para cada pregunta el número de respuestas varía, y, por tanto, 
TABLA I

Áreas temáticas

\begin{tabular}{l|l}
\hline \multicolumn{1}{c|}{ Área temática } & Campo principal de investigación declarado en la encuesta \\
\hline Medicina & Medicina, Odontología y Temas Relacionados. \\
\hline \multirow{2}{*}{ Física y Astronomía } & $\begin{array}{l}\text { Física y Ciencias Relacionadas y Astronomía y Ciencias del } \\
\text { Espacio. }\end{array}$ \\
\hline Agricultura y Ciencias Biológicas & Agricultura y Ciencias Relacionadas y Ciencias Biológicas. \\
\hline \multirow{2}{*}{ Ciencias Sociales y Humanidades } & $\begin{array}{l}\text { Negocios y Administración; Artes Creativas y Diseño; Edu- } \\
\text { cación; Estudios Históricos y Filosóficos, Lengua y Literatu- } \\
\text { ra; Leyes; Comunicación Masiva y Documentación; Psicolo- } \\
\text { gía y Ciencias Sociales. }\end{array}$ \\
\hline
\end{tabular}

se ha indicado en cada caso la cantidad de respuestas sobre las que se realizó el análisis de los datos.

\section{Resultados}

\section{Conocimiento y actitudes en relación con las revistas de acceso abierto}

El 73\% de los investigadores en las cuatro áreas analizadas, en su conjunto, dicen conocer la existencia de revistas que publican artículos en acceso abierto en sus respectivos campos, siendo más alto el porcentaje de respuestas positivas en Agricultura y Ciencias Biológicas (84\%) y en Medicina (81\%) que en Física y Astronomía (68\%) y Ciencias Sociales y Humanidades (61\%) (figura 1). Asimismo, el 94\% de los investigadores expresa que la publicación de artículos en revistas AA sería beneficioso para su campo de investigación, siendo el porcentaje muy similar en los cuatro campos (figura 2).

Entre las tres razones destacadas por las que los investigadores argentinos argumentan que las publicaciones en acceso abierto son positivas se encuentran: beneficio para la comunidad científica (41\%), razones financieras (25\%) y bien público (18\%); siendo estas motivaciones las más importantes en los cuatro campos de conocimiento analizados (figura 3).

\section{Influencia del acceso abierto en la decisión sobre dónde publicar}

Como se puede apreciar en la tabla II el prestigio de las revistas es, en todos los campos, el factor más importante que los investigadores tienen en cuenta a la hora de elegir las revistas donde publicar. También tienen un importante peso otros factores; todos ellos académicos.

El acceso abierto es considerado un aspecto importante para el $42 \%$ de los investigadores argentinos. Sin embargo, su peso no es igual en todos los campos, siendo más alto en Medicina y en Ciencias Sociales y Humanidades que en los otros. 
FIGURA 1

Conocimiento de la existencia de revistas que publican en acceso abierto

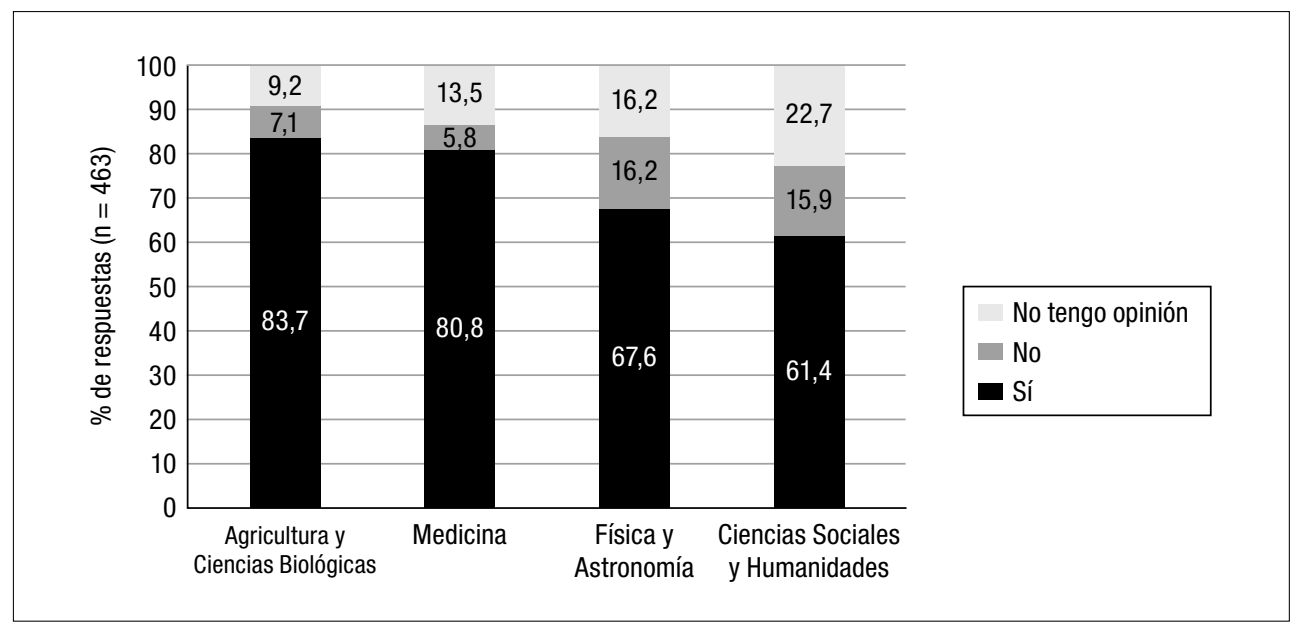

FIGURA 2

Acuerdo con la práctica de publicación en acceso abierto

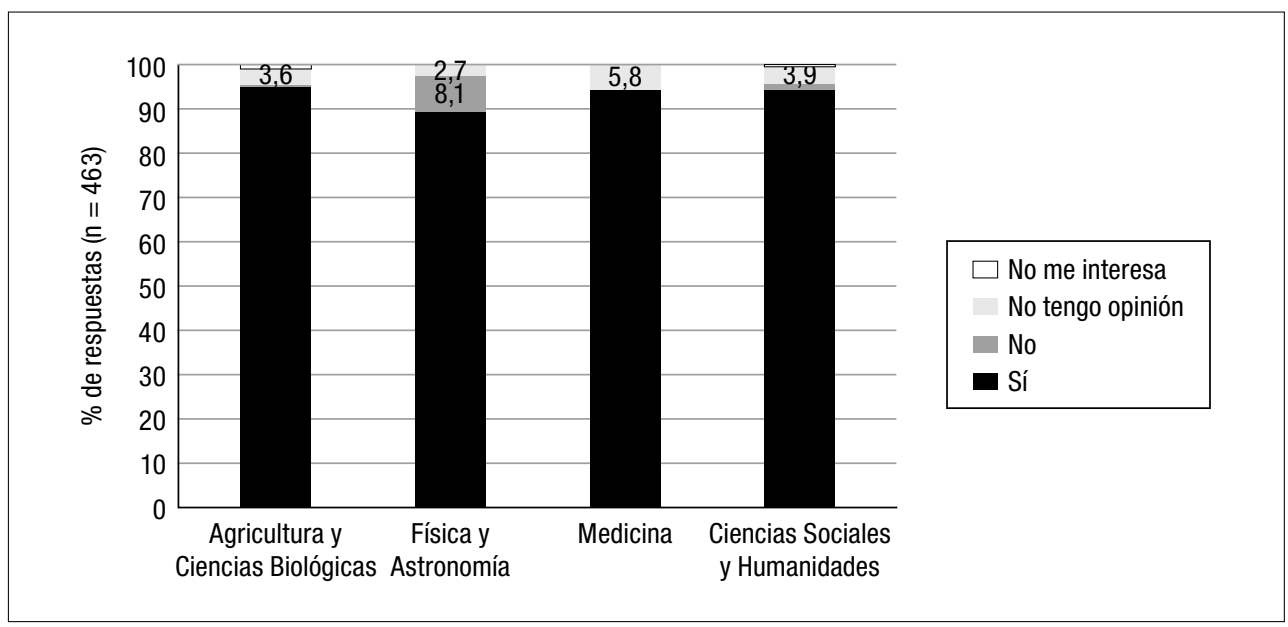

El $85 \%$ de los investigadores $(n=452)$ expresan haber publicado artículos en acceso abierto en los últimos cinco años, siendo Física y Astronomía el campo que reúne el más alto porcentaje de respuestas en las categorías 6 a 10 artículos o más de 10, con bastante diferencia respecto de los otros campos, cuyos porcentajes más altos se dan para la frecuencia 1 a 5 . Por otra parte, de los que declaran no haber publicado en AA en el período el más alto porcentaje (24\%) 
FIGURA 3

Percepción de los beneficios más importantes de publicar en abierto
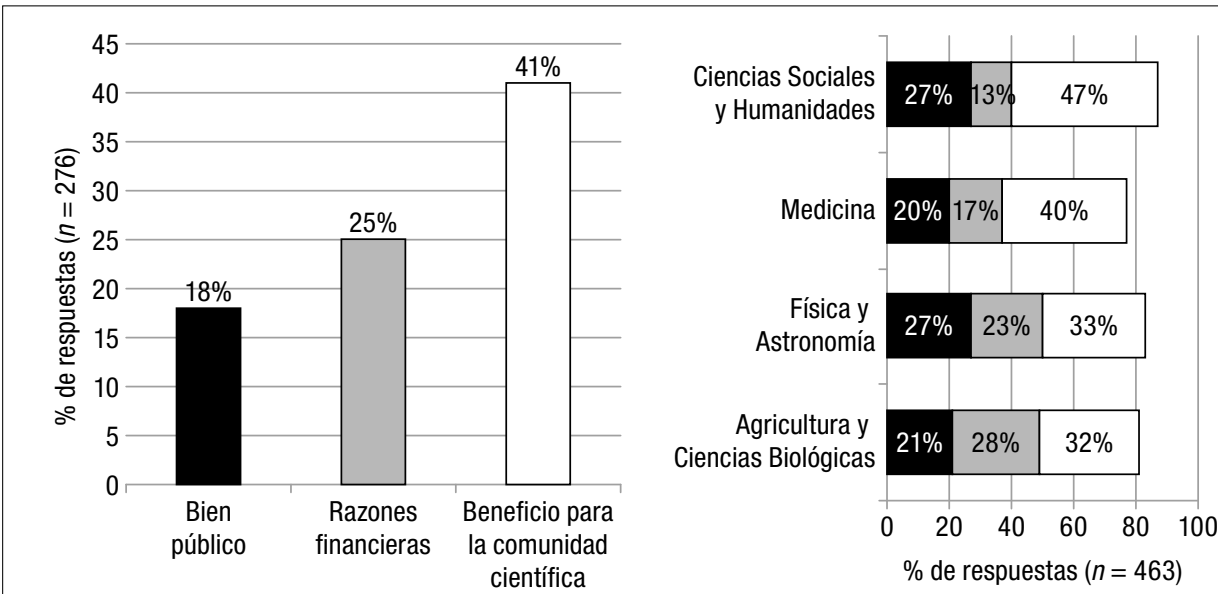

TABLA II

Factores que influyen en la elección de la revista donde publicar ( $n=358)$

\begin{tabular}{|c|c|c|c|c|c|}
\hline Factores & & $\begin{array}{l}\text { Agricultura } \\
\text { y Ciencias } \\
\text { Biológicas }\end{array}$ & $\begin{array}{c}\text { Física y } \\
\text { Astronomía }\end{array}$ & Medicina & $\begin{array}{c}\text { Ciencias } \\
\text { Sociales y } \\
\text { Humanidades }\end{array}$ \\
\hline Prestigio & $\begin{array}{l}\text { Sí } \\
\text { No }\end{array}$ & $\begin{array}{r}95,3 \\
4,7\end{array}$ & $\begin{array}{l}88,6 \\
11,4\end{array}$ & $\begin{array}{r}95,8 \\
4,2\end{array}$ & $\begin{array}{r}95,5 \\
4,5\end{array}$ \\
\hline Relevancia comunidad & $\begin{array}{l}\text { Sí } \\
\text { No }\end{array}$ & $\begin{array}{l}82,8 \\
17,2\end{array}$ & $\begin{array}{r}91,2 \\
8,8\end{array}$ & $\begin{array}{l}80,4 \\
19,6\end{array}$ & $\begin{array}{l}89,3 \\
10,7\end{array}$ \\
\hline Factor impacto & $\begin{array}{l}\text { Sí } \\
\text { No }\end{array}$ & $\begin{array}{r}92,2 \\
7,8 \\
\end{array}$ & $\begin{array}{l}75,0 \\
25,0\end{array}$ & $\begin{array}{l}76,1 \\
23,9\end{array}$ & $\begin{array}{l}63,8 \\
36,2 \\
\end{array}$ \\
\hline Gratuidad & $\begin{array}{l}\text { Sí } \\
\text { No }\end{array}$ & $\begin{array}{l}84,0 \\
16,0\end{array}$ & $\begin{array}{l}79,4 \\
20,6\end{array}$ & $\begin{array}{l}80,9 \\
19,1\end{array}$ & $\begin{array}{l}61,4 \\
38,6\end{array}$ \\
\hline Prob. de aceptación & $\begin{array}{c}\text { Sí } \\
\text { No }\end{array}$ & $\begin{array}{l}79,4 \\
20,6 \\
\end{array}$ & $\begin{array}{l}50,0 \\
50,0 \\
\end{array}$ & $\begin{array}{l}80,9 \\
19,1 \\
\end{array}$ & $\begin{array}{l}70,9 \\
29,1\end{array}$ \\
\hline Experiencia positiva & $\begin{array}{c}\text { Sí } \\
\text { No }\end{array}$ & $\begin{array}{l}74,2 \\
25,8 \\
\end{array}$ & $\begin{array}{l}64,7 \\
35,3\end{array}$ & $\begin{array}{l}89,1 \\
10,9 \\
\end{array}$ & $\begin{array}{l}64,9 \\
35,1\end{array}$ \\
\hline Velocidad de publicación & $\begin{array}{l}\text { Sí } \\
\text { No }\end{array}$ & $\begin{array}{l}85,3 \\
14,7\end{array}$ & $\begin{array}{l}63,6 \\
36,4\end{array}$ & $\begin{array}{l}63,6 \\
37,0\end{array}$ & $\begin{array}{l}58,0 \\
42,0\end{array}$ \\
\hline Recomendado colegas & $\begin{array}{l}\text { Sí } \\
\text { No }\end{array}$ & $\begin{array}{l}46,4 \\
53,6\end{array}$ & $\begin{array}{l}53,1 \\
46,9\end{array}$ & $\begin{array}{l}61,7 \\
38,3\end{array}$ & $\begin{array}{l}70,9 \\
29,1\end{array}$ \\
\hline Acceso abierto & $\begin{array}{c}\text { Sí } \\
\text { No }\end{array}$ & $\begin{array}{l}52,9 \\
47,1 \\
\end{array}$ & $\begin{array}{l}45,5 \\
54,5 \\
\end{array}$ & $\begin{array}{l}68,2 \\
31,8 \\
\end{array}$ & $\begin{array}{l}62,8 \\
37,2 \\
\end{array}$ \\
\hline Política institucional & $\begin{array}{c}\text { Sí } \\
\text { No }\end{array}$ & $\begin{array}{l}25,8 \\
74,2\end{array}$ & $\begin{array}{l}16,1 \\
83,9\end{array}$ & $\begin{array}{l}42,2 \\
57,8\end{array}$ & $\begin{array}{l}35,8 \\
64,2\end{array}$ \\
\hline
\end{tabular}


se da en Ciencias Sociales y Humanidades, siendo muy inferior en los otros campos (figura 4).

Al relacionar las variables de prestigio de la revista y frecuencia de publicación en abierto en los últimos cinco años se encontró una relación positiva, ya que un alto porcentaje de investigadores que señalan haber publicado en AA consideran el prestigio como un factor muy importante en la elección de las revistas donde publicar. El porcentaje es del orden del 66\% en Ciencias Sociales y Humanidades y superior al 83\% en los otros tres campos.

\section{FIGURA 4}

Cantidad de artículos publicados en abierto en los últimos cinco años

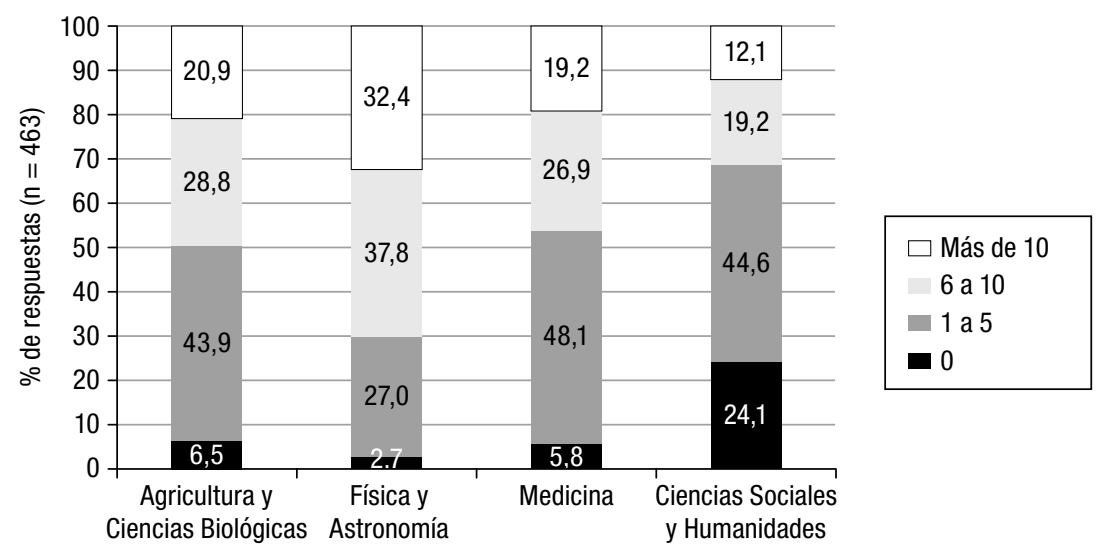

FIGURA 5

Razones para no publicar en abierto $(n=38)$

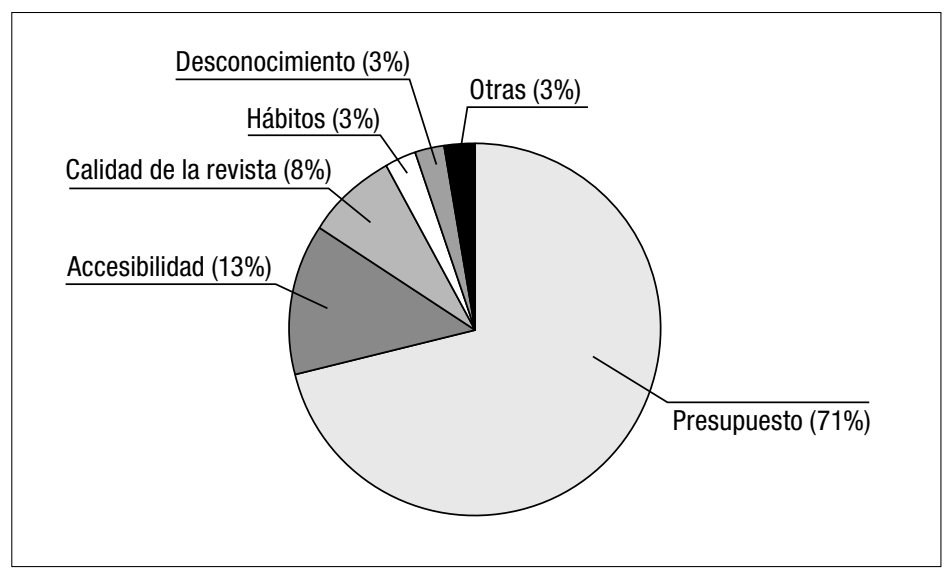


Solo hubo 38 respuestas que especificaron razones para no publicar en abierto. El $71 \%$ de este grupo señaló como primer motivo cuestiones presupuestarias, mientras que otras razones como accesibilidad, calidad, hábitos, desconocimiento u otros aparecen con muy menor presencia (figura 5).

\section{Discusión y conclusiones}

Los resultados de este estudio muestran que un alto porcentaje de los investigadores argentinos en las distintas áreas temáticas expresan conocer la existencia de revistas de acceso abierto en sus campos del conocimiento. Destacan las áreas de Agricultura y Ciencias Biológicas y Medicina. Comparando estos resultados con los obtenidos en el estudio a escala mundial (Dallmeier-Tiessen y otros (2011) se aprecia una coincidencia, aunque los porcentajes son levemente superiores en el caso argentino.

Este estudio muestra también coincidencias entre las opiniones de los investigadores argentinos y la comunidad internacional que participó de la encuesta SOAP, en lo referente a los tres aspectos más importantes por los que el acceso abierto es considerado beneficioso. Los porcentajes son mayores en el caso argentino a los hallados a nivel mundial en los dos primeros aspectos: por el bien de la comunidad científica y por razones financieras; en tanto que son similares en el tercero (beneficioso para el bien público). Esta situación se repite en las distintas áreas temáticas analizadas.

El porcentaje de investigadores argentinos que expresan haber publicado al menos un artículo en abierto en los últimos cinco años es mucho más alto que el hallado a escala mundial ( $85 \%$ vs $52 \%$ ). Los porcentajes son mayores en Física y Astronomía y menores en Ciencias Sociales y Humanidades, siendo esta última la que registra la mayor proporción de respuestas en la categoría 0 artículos. Lamentablemente en el trabajo de Dallmeier-Tiessen y otros (2011) no se incluyen resultados de este aspecto desagregados por disciplinas, motivo por el cual no es posible determinar si las diferencias observadas entre áreas temáticas se deben a un comportamiento de los investigadores argentinos o es una característica también presente en la comunidad internacional.

Los resultados de este estudio muestran también que aunque es muy bajo el porcentaje de investigadores argentinos que señalan razones para no publicar en abierto, se observa una coincidencia con los resultados encontrados a escala mundial en el que los costos por publicación y la baja calidad de las revistas aparecen como las dos principales barreras de la vía dorada del AA.

Además, en concordancia con los hallazgos a nivel mundial (Dallmeier-Tiessen y otros, 2011) y los resultados de otros estudios previos (Mann y otros, 2009; Harley y otros, 2010; Kenney, y Warden, R., 2011; Miguel y otros, 2011a, b), este trabajo confirma que es el prestigio de las revistas el factor más importante que los investigadores tienen en cuenta a la hora de elegir las revistas donde publicar sus trabajos, siendo el acceso abierto un aspecto de relevancia menor a aquel en todos los campos temáticos. 
Por otra parte, al contrastar las opiniones de los investigadores argentinos con los resultados del estudio de las prácticas de publicación según el modelo de acceso de las revistas realizado por Miguel y otros (en prensa) se encontraron las siguientes discrepancias: mientras los resultados del presente estudio revelan que un alto porcentaje de investigadores expresa conocer revistas AA en sus respectivos campos, y lo considera beneficioso, el estudio de las prácticas muestra que la mayoría de los artículos son publicados en revistas de acceso restringido, que permiten con o sin condiciones el auto-archivo de una copia del trabajo en repositorios institucionales o temáticos.

En particular, hay dos áreas que presentan una mayor discrepancia entre opiniones y prácticas, Ciencias Sociales y Humanidades y Física y Astronomía:

- En el primer caso (Ciencias Sociales y Humanidades), mientras los resultados de la encuesta SOAP muestran que es el área con menor porcentaje de trabajos publicados en AA en los últimos cinco años; el estudio de las prácticas revela que es el campo con mayor porcentaje de artículos publicados en revistas de la vía dorada.

- En el segundo caso (Física y Astronomía) sucede lo contrario. Mientras el estudio de las prácticas revela que es el campo con menor porcentaje de artículos publicados en revistas AA, es el campo con el más alto porcentaje de investigadores que dicen haber publicado artículos en acceso abierto en los últimos cinco años.

Si bien es posible que las diferencias observadas entre el estudio de opiniones del caso argentino con los hallazgos a nivel mundial, así como las discrepancias entre las opiniones y prácticas puedan vincularse con sesgos producidos tanto por el tamaño de la muestra de los investigadores que contestaron la encuesta SOAP, como por limitaciones en la cobertura de la fuente de datos utilizada en el estudio de Miguel y otros (en prensa), también podrían tener otras explicaciones que se exponen a continuación.

Por un lado, la superioridad de los porcentajes del caso argentino respecto de los hallazgos a nivel mundial con relación a las opiniones sobre el AA podrían deberse a las políticas y acciones para la adopción de modelos de acceso abierto que están teniendo lugar en Argentina. Si bien en dicho país no existen subsidios específicos que financien la publicación de artículos en acceso abierto en revistas internacionales, sí se impulsan publicaciones científicas nacionales en acceso abierto, tales como la representación argentina de Scientific Electronic Library Online (SciELO), www.scielo.org.ar, parte de una red iberoamericana de colecciones de revistas científicas en acceso abierto y el Portal de Publicaciones Científicas y Técnicas (PPCT), ppct.caicyt.gov.ar, ambos proyectos financiados por el Consejo Nacional de Investigaciones Científicas y Técnicas (CONICET) y gestionados a través del Centro Argentino de Información Científica y Tecnológica (CAICYT, 2011).

Por otro lado se promueve también el acceso abierto a través de la vía verde del AA, con un fuerte protagonismo del Ministerio de Ciencia, Tecnología e Innovación Productiva de la Nación (MINCYT) y de las Universidades Nacionales, que 
son las responsables de más del $80 \%$ de la producción científica del país. En este sentido, el MINCyT ha creado el Sistema Nacional de Repositorios Digitales (SNRD) e impulsado un proyecto de ley para el auto-archivo de la producción científicotecnológica que sea resultado de la realización de actividades de investigación financiadas con fondos públicos (Argentina, 2011).

Por otra parte, las diferencias halladas para el caso argentino entre opiniones y prácticas, especialmente en los campos de las Ciencias Sociales y Humanidades y en Física y Astronomía podrían deberse a las distintas apreciaciones sobre lo que significa la comunicación de artículos en AA en cada disciplina. De acuerdo a los recientes hallazgos de Fry y otros (2011) los investigadores en Física y en Ciencias Sociales, Artes y Humanidades relacionan el acceso abierto más con el auto-archivo (vía verde) que con la publicación en revistas de la vía dorada, contrariamente a lo percibido en Ciencias Médicas y Biología. Por tanto, aunque la encuesta SOAP haya estado dirigida al conocimiento de opiniones en relación a la vía dorada del AA no es posible saber cómo han interpretado el acceso abierto los investigadores participantes, lo que podría explicar esta disociación.

Las discrepancias observadas también podrían vincularse a una suerte de contradicción que pareciera existir entre una inclinación a favor del AA (desde las opiniones) y cierta cautela en el cambio de hábitos (prácticas), motivadas por el temor por parte de los investigadores a no recibir evaluaciones favorables que les permitan avanzar en sus carreras académicas por publicar en revistas AA de baja calidad, tal como señala el estudio de Mann y otros (2009). Esto se reafirma con las conclusiones del estudio del proyecto PEER que señalan que los investigadores tienen actitudes, percepciones y comportamientos conservadores hacia el sistema de comunicación académica y no buscan cambios estructurales en la difusión y publicación de los resultados de investigación (Fry y otros, 2011).

En el caso argentino fueron muy pocos los que expusieron razones para no publicar en abierto; sin embargo, de los que respondieron a esta pregunta la mayoría señaló barreras económicas como problema principal.

A partir de las interpretaciones realizadas se podría inferir que, además de las tradiciones y de las percepciones que sobre el AA tienen los investigadores en cada campo disciplinar, el modelo de pago por publicación y la desconfianza en la calidad de las revistas de acceso abierto parecen ser dos factores claves en las posibilidades de desarrollo de la vía dorada, que se suman al limitante de su escasa cantidad respecto del total de revistas que se editan a nivel mundial (Laakso y otros, 2011).

A modo de conclusión final, este estudio da cuenta de que aunque a los científicos la idea del acceso abierto les parece atractiva y beneficiosa, eso no es suficiente para cambiar sus hábitos de publicación. Es evidente que hay mucho trabajo por hacer desde los editores, los organismos de financiamiento, las instituciones y los gestores de información, para ofrecer a los autores opciones donde los beneficios de publicar en abierto sean visibles y ampliamente reconocidos por los pares y evaluadores.

Por último agregar, que es necesario continuar en la línea de estudios que contrasten opiniones y prácticas de comunicación en acceso abierto, tanto por 
la vía dorada como por la vía verde, para incrementar el conocimiento actualmente existente y para impulsar acciones en pos de su desarrollo en función de las tradiciones y prácticas de cada campo de conocimiento.

\section{Agradecimientos}

A María Luisa Lascuraín Sánchez por sus comentarios y sugerencias.

Este estudio ha sido realizado en el marco de los proyectos:

(H540) «El acceso abierto al conocimiento científico en Argentina. Estado de la cuestión y principales tendencias». Programa de Incentivos a Docentes Investigadores de las Universidades Nacionales, Ministerio de Educación, Argentina.

«Hacia un modelo teórico, metodológico y tecnológico para el repositorio institucional de acceso abierto de la UNR fundamentado en las prácticas de su comunidad académica» - Universidad Nacional de Rosario, CIFASIS (UNR-CONICET-UPCM). Argentina

\section{Bibliografía}

Argentina. Honorable Cámara de Diputados de la Nación (2011). Proyecto de Ley 1927D-2011 Creación de Repositorios Digitales Institucionales de Acceso Abierto, Propios o Compartidos. Disponible en: http://www1.hcdn.gov.ar/proyxml/expediente. asp?fundamentos=si\&numexp=1927-D-2011 [consulta: 21/10/2011].

Björk, B. C.; Welling, P.; Laakso, M.; Majlender, P.; Hedlund, T., y Guðnason, G. (2010): Open access to the scientific journal literature: Situation 2009. Plos One, 5 (6). Disponible en http://www.plosone.org/article/info:doi/10.1371/journal.pone.0011273 [consulta: 18/08/2011].

Budapest Open Access Initiative (2001): Budapest, Hungary: Open Society Institute. http:// www.soros.org/openaccess/read.shtml [consulta: 18/08/2011].

CAICYT-CONICET (2011): SciELO Argentina y desarrollos institucionales. En: Necobelac T1 - Resultados de la investigación en ciencias de la salud: el proceso de publicación y el acceso abierto. Buenos Aires, Argentina, 16-18 Mayo. Disponible en http://www. necobelac.eu/documents/BA/ExperienciasLocales_SciELO-Argentina \%20CAICYT.ppt [consulta: 26 de octubre de 2011].

Dallmeier-Tiessen, S.; Darby, R.: Goerner, B.; Hyppoelae, J.; Igo-Kemenes, P., y Kahn, D. (2011): Highlights from the SOAP project survey. What scientists think about open access publishing. Disponible en http://arxiv.org/abs/1101.5260 [consulta: 13-08-2011].

Fry, J.; Probets, S.; Creaser, C.; Greenwood, H.; Spezi, V., y White, S. (2011): PEER Behavioural Research: Authors and Users vis-à-vis Journals and Repositories. Final Report. Loughborough: Loughborough University, Department of Information Science; LISU. Disponible en: http://www.peerproject.eu/fileadmin/media/reports/PEER_D4_final_ report_29SEPT11.pdf [consulta: 16/08/2011].

Gómez, N.; Bustos-Gonzalez, A., y Muñoz, G. (2008): Los nuevos canales de comunicación de la ciencia y la respuesta de los científicos chilenos. En Seminario nuevas tendencias en información y sus implicancias en el desarrollo profesional bibliotecario, San- 
tiago, Chile. Disponible en http://eprints.rclis.org/bitstream/10760/14635/1/Los_nuevos_canales_de_comunicaci\%c3\%b3n_de_la_ciencia4.pdf [consulta: 18/08/2011].

Harley, D.; Acord, S. K.; Earl-Novell, S.; Lawrence, S., y King, C. J. (2010): Assessing the future landscape of scholarly communication: An exploration of faculty values and needs in seven disciplines. Disponible en http://escholarship.org/uc/cshe_fsdc [consulta: 20/08/2011].

Harnad, S.; Brody, T.; Vallieres, F.; Carr, L.; Hitchcock, S., y Gingras, Y. (2008): The access/ impact problem and the green and gold roads to open access: An update. Serials Review, 34(1), 36-40. Disponible en http://eprints.ecs.soton.ac.uk/15852/ [consulta: 18/08/2011].

Hurrell, C., y Meijer-Kline, K (2011): Open Access up for Review: Academic Attitudes towards Open Access Publishing in Relation to Tenure and Promotion. Open Excess: The Journal of Scholarly Communicators, 1 (3). Disponible en http://tsc.library.ubc. ca/index.php/journal4/article/view/104 [consulta: 18/10/2011].

Kenney, R., y Warden, R. (2011): An Open Access future? Report from the eurocancercoms project. Ecancer 5(223). Disponible en http://ecancer.org/ecms/4/203/full [consulta: 10/09/2011].

Laakso, M.; Welling, P.; Bukvova, H.; Nyman, L.; Bjork, B., y Hedlund, T. (2011): The development of open access journal publishing from 1993 to 2009. Plos One, 6(6), e20961. Disponible en http://www.plosone.org/article/info:doi/10.1371/journal.pone.0020961 [consulta: 5/08/2011]

Mann, F.; Hess, T.; Von, W. B., y Wigand, R. T. (2009): Open access publishing in science. Communications of the Acm, 52, 3, 135-139.

Miguel, S. E.; Gómez, N. D., y Bongiovani, P. C. (2011a): El acceso abierto a las publicaciones de la comunidad científica argentina en el campo de la medicina. E-Colabora: Revista de Ciencia, Educación, Innovación y Cultura, 1(2). Disponible en http://publicaciones.renata.edu.co/index.php/RCEC/article/view/36 [consulta: 20/08/2011].

Miguel, S. E.; Gómez, N. D., y Bongiovani, P. C. (en prensa): Acceso abierto real y potencial a la producción científica de un país. El caso argentino. El profesional de la información (ene-feb 2012).

Miguel, S. E.; Chinchilla-Rodríguez, Z., y de Moya-Anegón, F. (2011b): Open access and Scopus: A new approach to scientific visibility from the standpoint of access. Journal of the American Society for Information Science and Technology, 62 (6), 1130-1145.

Ministerio de Ciencia, Tecnología e Innovación Productiva. Indicadores de Ciencia y Tecnología Argentina 2008 (2010): Ciudad Autónoma de Buenos Aires. Disponible en http://www.mincyt.gov.ar/multimedia/archivo/archivos/Indicadores_WEB4mb.pdf [consulta: 10/10/2011].

RIN; JISC; PRC; RLUK, y Wellcome Trust (2011): Heading for the Open Road: Costs and Benefits of Transitions in Scholarly Communications. Disponible en http://www.rin. ac.uk/our-work/communicating-and-disseminating-research/heading-open-road-costsand-benefits-transitions-s [consulta: 10/08/2011].

Sánchez-Tarragó, N., y Fernández-Molina, C. (2008): Conocimientos y actitudes de los investigadores cubanos de la salud hacia las revistas de acceso abierto. Acimed, 17 (3). Disponible en http://scielo.sld.cu/scielo.php?script=sci_arttext\&pid=\$1024-94352008000 300002\&lng=es\&nrm=iso [consulta: 20/08/2011].

SOAP survey data - Release Notes (2011): Disponible en http://bit.ly/gI8nct. [consulta: 10/08/2011]. 